\title{
Improving outcomes from ischaemic colitis: more questions than answers
}

\author{
Amy Gardiner • Aonghus McGivney • Patrick McGuire • \\ Raymond Oliphant $\cdot$ on behalf of the RAH Virtual \\ Journal Club
}

Accepted: 29 May 2014 / Published online: 6 June 2014

(C) Springer-Verlag Berlin Heidelberg 2014

\section{Dear Editor:}

We read with interest the recent paper by Genstorfer et al. [1] that described outcomes and risk factors for in-hospital mortality following surgery for ischaemic colitis. The authors draw attention to a common morbid surgical condition that is frequently fatal. We appreciate that such retrospective cohort studies have many weaknesses as acknowledged by the authors in their discussion. However, we would like to highlight a few significant limitations of this study that require further clarification.

First, we were disappointed to note that the presence of known risk factors of adverse surgical outcomes such as diabetes and patient smoking status were not recorded or included in this analysis. In particular, diabetic hyperglycaemia is associated with acidaemia, ketosis and impaired wound healing [2]. Given that reduced preoperative $\mathrm{pH}$ level was reported as an important risk factor for inhospital mortality, we found it surprising that the influence of this potential confounding factor was not discussed.

Second, the authors report that prior cardiac or aortic surgery was an independent risk factor for in-hospital mortality. However, this conclusion seems somewhat overstated as the results presented suggest, if anything, a weak non-significant association only (OR 2.4; $95 \%$ CI 0.82 to $6.8 ; P=0.109$ ). In addition, it is unclear why the authors presented postoperative death as "in-patient death" rather than the more conventional 30-day post-operative mortality. This makes comparison with previously published series difficult.

Finally, the finding that major post-operative surgical complications were more likely to occur in patients with histologically proven ischaemic changes at the resection margin appears to be at odds with the authors contradictory comment in the discussion that "primary anastomosis can be performed, when blood supply of the residual colon and the formed anastomosis seems to be sufficient in the eyes of an experienced surgeon". Therefore, it would be of interest if the authors could have measured whether the intraoperative assessment of colonic viability correlated well with subsequent histological evaluation.

We agree with the authors that larger, prospective, multicentre cohort studies are required to further evaluate predictors of mortality and surgical complications in patients undergoing surgery for ischaemic colitis. It is hoped that identification of clinically relevant factors will not only hasten the accurate diagnosis of ischaemic colitis but also ensure appropriate surgical management, with the aim of reducing the burden of morbidity and mortality associated with this condition.

Conflict of interest All authors declare no conflict of interests.

\section{References}

1. Genstorfer J, Schäfer J, Kettelhack C, Oertli D, Rosenthal R (2014) Surgery for ischaemic colitis: outcome and risk factors for in-hospital mortality. Int J Color Dis 29:493-503. doi:10.1007/s00384-0131819-1

2. Hanazaki K, Maeda H, Okabayashi T (2009) Relationship between perioperative glycaemic control and postoperative infections. World J Gastroenterol 15(33):4122-4125. doi:10.3748/wjg.15.4122

\footnotetext{
A. Gardiner $\cdot$ A. McGivney $\cdot$ P. McGuire $\cdot$ R. Oliphant $(\bowtie)$

Department of General Surgery, Royal Alexandra Hospital, Corsebar

Road, Paisley, Scotland PA2 9PN, UK

e-mail: raymondoliphant@nhs.net
} 